\title{
Two Ocular Angiostrongyliasis Cases in Thailand with Molecular Identification of Causative Parasite Species
}

\author{
Patcharaporn Boonroumkaew, ${ }^{1,2}$ Thuss Sanguansak, ${ }^{3}$ Siriraksa Visaetsilpanonta, ${ }^{4}$ Oranuch Sanpool, ${ }^{1,2}$ Lakkhana Sadaow, ${ }^{1,2}$ \\ Pewpan M. Intapan, ${ }^{1,2}$ and Wanchai Maleewong ${ }^{1,2 *}$ \\ ${ }^{1}$ Department of Parasitology, Faculty of Medicine, Khon Kaen University, Khon Kaen, Thailand; ${ }^{2}$ Research and Diagnostic Center for Emerging \\ Infectious Diseases, Faculty of Medicine, Khon Kaen University, Khon Kaen, Thailand; ${ }^{3}$ Department of Ophthalmology, Faculty of Medicine, Khon \\ Kaen University, Khon Kaen, Thailand; ${ }^{4}$ Department of Ophthalmology, Retina Unit, Udon Thani Hospital, Udon Thani, Thailand
}

\begin{abstract}
Human angiostrongyliasis is an important foodborne helminthic zoonosis caused by the nematodes of the genus Angiostrongylus. We describe two parasitologically confirmed cases of ocular angiostrongyliasis, presenting at a tertiary care hospital in Thailand between 2012 and 2018. Parasites were surgically recovered from patients' eyes and morphologically identified as Angiostrongylus species. DNA analysis allowed identification of Angiostrongylus cantonensis. Polymerase chain reaction was used to amplify all or part of the small nuclear ribosomal subunit, the second internal transcribed spacer region, and cytochrome c oxidase subunit 1 . The sequences subsequently obtained were highly similar to those of $A$. cantonensis (97-100\%). This is the first molecular confirmation that $A$. cantonensis is a causative agent of human angiostrongyliasis in Thailand.
\end{abstract}

\section{INTRODUCTION}

Angiostrongyliasis is a foodborne nematode disease caused by members of the genus Angiostrongylus. These are normally parasites of rodents and carnivores, in which they live in the mesenteric arteries or the pulmonary arteries and lungs. ${ }^{1}$ Angiostrongylus cantonensis, a rat lungworm, is a primary cause of human eosinophilic meningitis or meningoencephalitis in many countries, and more than 2,800 cases have been documented worldwide. ${ }^{2-6}$ Humans are accidental hosts who become infected by consuming snails, slugs, and paratenic hosts containing, or vegetables contaminated with infective larvae. ${ }^{4,7}$ Angiostrongylus costaricensis is the cause of abdominal angiostrongyliasis in Latin American countries. ${ }^{8}$ Angiostrongylus mackerrasae and Angiostrongylus malaysiensis are two other species that may cause harm in some people ${ }^{9}$ and possibly cause the disease in this area. Morphological identification of the causative Angiostrongylus species is difficult because of the overlap and similar descriptions of size and body shapes among different species. ${ }^{1,10,11}$ The confirmed species by the molecular test should call attention from clinicians dealing with neurological patients to be aware of the causative agents of infection.

Although the incidence of ocular angiostrongyliasis is low, it can cause optic neuritis resulting in vision loss, which can seriously lower the patient's life quality. ${ }^{12}$ Until now, at least 44 ocular cases have been reported worldwide, nearly half of these were from Thailand and the rest from other Asian countries, such as Sri Lanka, India, Taiwan, Vietnam, Malaysia, Japan, and Indonesia, ${ }^{12,13}$ and from South America. ${ }^{14}$ Recently, 18 ocular angiostrongyliasis cases presenting between the years 1995 and 2017 were retrospectively studied in Thailand. ${ }^{15}$ To our knowledge, no information has been published on molecular identification of Angiostongylus species recovered from patients in Thailand. In this study, DNA sequences from portions of the nuclear small ribosomal subunit (SSU), the second internal transcribed spacer region (ITS2),

\footnotetext{
*Address correspondence to Wanchai Maleewong, Department of Parasitology, Faculty of Medicine, Khon Kaen University, 123 Mitraparp Rd., Amphur Muang, Khon Kaen 40002, Thailand. E-mail: wanch_ma@kku.ac.th
}

and the cytochrome $\mathrm{c}$ oxidase subunit 1 (COI) gene were obtained from worms surgically removed from two ocular angiostrongyliasis patients. The sequences were identified as being from $A$. cantonensis. The phylogenetic relationships between $A$. cantonensis and other Angiostrongylus species are also discussed.

\section{MATERIALS AND METHODS}

Patients and specimens. In each of the years 2012 (patient 1) and 2018 (patient 2), an Angiostrongylus worm was surgically recovered from the anterior chamber of the left eye of a patient. Both patients were diagnosed as angiostrongyliasis cases by clinical signs and symptoms (Figures 1 and 2) at Srinagarind Hospital, Khon Kaen University, Khon Kaen, and at the Provincial Hospital, Udon Thani, Thailand, respectively. Briefly, patient 1, a 50-year-old man, presented with body pain, headache, eosinophilic meningitis, and astigmatism. The incubation period was 2 weeks. The size (length $\times$ width) of recovered worm (fifth-stage male larva) was $8 \times 0.5 \mathrm{~mm}$. Patient 2, a 70-year-old man, presented with astigmatism. The incubation period was 1 week. The size (length $\times$ width) of recovered worm (fifth-stage female larva) was $10 \times 0.25 \mathrm{~mm}$. The recovered worms were kept in $70 \%$ ethanol and stored at $-70^{\circ} \mathrm{C}$ at Srinagarind Hospital, Khon Kaen University until use for DNA extraction. Both patients made a full recovery. The authors have complied with all the ethics guidelines applicable. This study was approved by the Khon Kaen University Ethics Committee for Human Research (HE621018).

DNA extraction, polymerase chain reaction (PCR) amplification, DNA sequencing, and sequence analysis. Genomic DNA was extracted from a part of each worm using the NucleoSpin ${ }^{\circledR}$ tissue kit (Macherey-Nagel GmbH \& Co. KG, Duren, Germany) according to the manufacturer's instructions. Genomic DNAs were further subjected to polymerase chain reaction using SSU rRNA primers; Angi18S-1_forward: 5'-AAAGTTAAGCCATGCATG-3' and Angi18S-2_reverse: 5' CATTCTTGGCAAATGCTTTCG- ${ }^{\prime},{ }^{16}$ COI primers; AngiCOI_forward: 5'-TाTाTTGGGCATCCTGAGGTTTAT-3' and AngiCOI_reverse: 5'-CGAGGATAACCATGTAAACCAGC-3' ${ }^{\prime 17}$; and ITS2 primers; AngilTS2_forward: 5'-ACATCTGGTTCA GGGTTGTT-3' and AngilTS2_reverse: 5'-AGCATACAAGC 


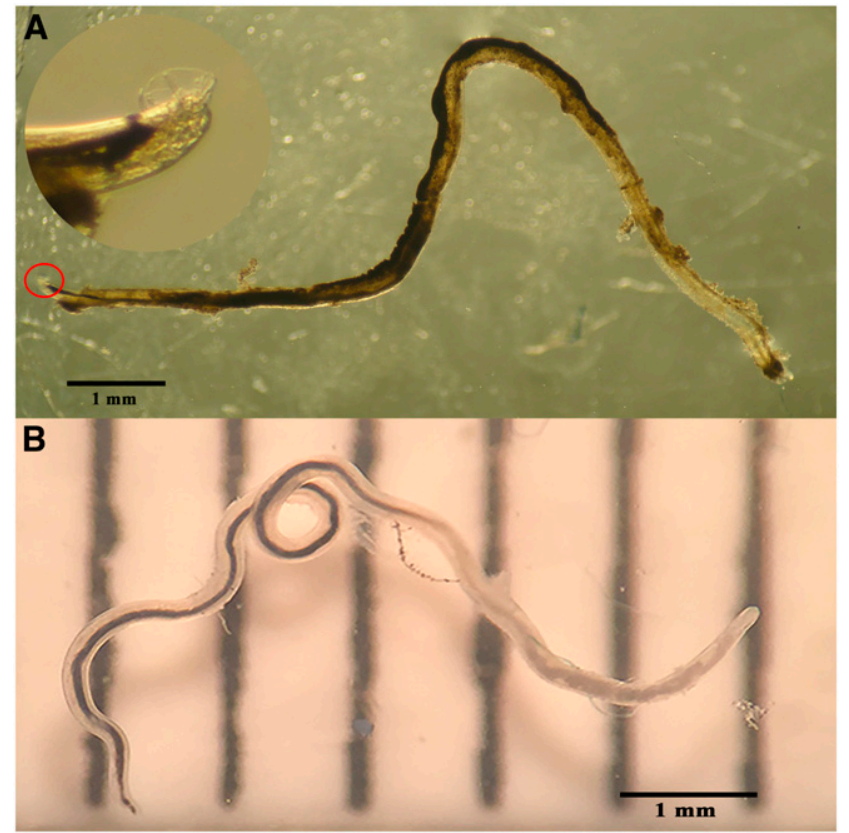

FIGURE 1. (A) Angiostrongylus cantonensis fifth-stage larva recovered from the anterior chamber of the left eye of patient 1 . A circle indicates the enlargement of the posterior end of the worm showing the small single-lobed bursa characteristic of a male. (B) Fifth-stage larva of $A$. cantonensis recovered from the anterior chamber of the left eye of patient 2. This figure appears in color at www.ajtmh.org.

ACATGATCAC-3'. ${ }^{17}$ Each PCR contained $0.5 \mu \mathrm{L}$ of each primer $(10 \mu \mathrm{M}), 2.5 \mu \mathrm{L}$ of $10 \times$ FastStart High Fidelity Reaction Buffer without $\mathrm{MgCl}_{2}$ (Roche, Mannheim, Germany), $2 \mu \mathrm{L}$ of $\mathrm{MgCl}_{2}(1.8 \mathrm{mM}), 0.5 \mu \mathrm{L}$ of dNTP mix (10 mM), 0.125 units of FastStart High Fidelity Enzyme Blend (Roche), and $2.5 \mu \mathrm{L}$ of DNA template from individual worms. The volume was adjusted to $25 \mu \mathrm{L}$ using sterile deionized water. For amplification of SSU rRNA or COI DNA, each reaction was preincubated for 2 minutes at $94^{\circ} \mathrm{C}$; followed by 10 cycles at $94^{\circ} \mathrm{C}$ for 1 minute (denaturation), $40^{\circ} \mathrm{C}$ for 1 minute (annealing), and $68^{\circ} \mathrm{C}$ for 2 minutes (extension); and then 30 cycles at $94^{\circ} \mathrm{C}$ for 1 minute (denaturation), $45^{\circ} \mathrm{C}$ for 1 minute (annealing), $68^{\circ} \mathrm{C}$ for 2 minutes (extension), and final extension at $68^{\circ} \mathrm{C}$ for 7 minutes. ${ }^{17}$ For ITS2

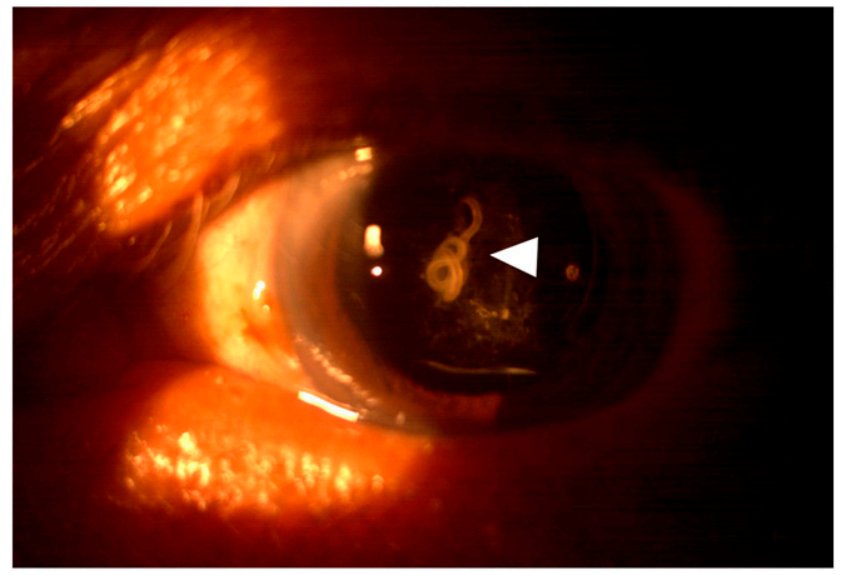

Figure 2. Angiostrongylus cantonensis fifth-stage larva in the anterior chamber of the left eye of patient 2 . This figure appears in color at www.ajtmh.org.
DNA amplification, the reaction was preincubated for 5 minutes at $94^{\circ} \mathrm{C}$; followed by 35 cycles at $94^{\circ} \mathrm{C}$ for 30 seconds (denaturation), $55^{\circ} \mathrm{C}$ for 30 seconds (annealing), and $72^{\circ} \mathrm{C}$ for 30 seconds (extension); and final extension at $72^{\circ} \mathrm{C}$ for 7 minutes. ${ }^{17}$ Amplicons were run on a $1.0 \%(\mathrm{w} / \mathrm{v})$ agarose gel and stained with ethidium bromide to visualize the amplified PCR product under ultraviolet illumination. The PCR products were then subjected to DNA sequencing in both directions using PCR primers as sequencing primers (Apical Scientific Sdn Bhd, Selangor, Malaysia). The sequences were aligned and compared with representative nucleotide sequences of Angiostrongylus species from GenBank using the multiple sequence alignment program ClustalW within BioEdit Sequence Alignment Editor version 7.0.9 (Tom Hall, North Carolina State University, Raleigh, NC). ${ }^{18}$ Similarity searches against known Angiostrongylus species sequences were performed using the Basic Local Alignment Search Tool at the National Center for Biotechnology Information website (https://blast.ncbi.nlm.nih.gov/Blast.cgi) (see GenBank accession numbers of selected reference sequences in the following text). All sequences generated in this study were deposited in the GenBank database under the following accession numbers: MK508859-MK508860 for the SSU rRNA region, MK512070-MK512071 for the ITS2 region, and MK516735-MK516736 for the COI gene.

Phylogenetic trees were constructed using the maximum likelihood (ML) and Bayesian inference $(\mathrm{BI})$ methods executed in MEGA version 7.0 (Sudhir Kumar, Arizona State University, Tempe, AZ) ${ }^{19}$ and MrBayes version 3.4 software, $^{20}$ respectively. The best model for SSU rRNA and ITS2 regions was the Tamura 3-parameter model (T92) and for the COI gene, it was the Tamura-Nei model (TN93) with nonuniformity of evolutionary rates among sites using a distinct gamma distribution (+G). Support for clusters in the ML tree was calculated using 1,000 bootstrap replications. For $\mathrm{Bl}$ analyses, the SSU rRNA, ITS2, and COI alignments were run for one million six hundred thousand, seven hundred thousand, and one million generations, respectively (two runs, each of four chains), by which time the SD of split frequencies had decreased less than 0.01 . Trees were sampled every 1,000 generations. Examination of the output (using the "sump" command) indicated that the potential scale reduction factor was 1 for relevant parameters and that stationarity had been approached after $25 \%$ of generations, and the first $25 \%$ of trees had been discarded as burnin.

\section{RESULTS}

A single worm was obtained from each of two patients with ocular angiostrongyliasis. The partial SSU rRNA gene (885 bp), partial ITS2 region (395 bp), and partial COI gene (605 bp) were successfully amplified from the genomic DNA of both worms and sequenced. After trimming the primer sequences, all sequences from the present study were compared with $A$. cantonensis sequences in GenBank using phylogenetic trees (Figures 3-5). Within $A$. cantonensis, the SSU rRNA sequence exhibited seven variable sites $(0.8 \%)$ in the 846-bp alignment. The ITS2 sequences exhibited eight variable sites $(2.3 \%)$ in the $356-b p$ alignment, and the $\mathrm{COI}$ sequences exhibited 31 variable sites (5.6\%) in the 558-bp alignment. All sequences from our worms were located in the same clade as reference $A$. cantonensis sequences, thus confirming their identity as $A$. cantonensis. Trees constructed 


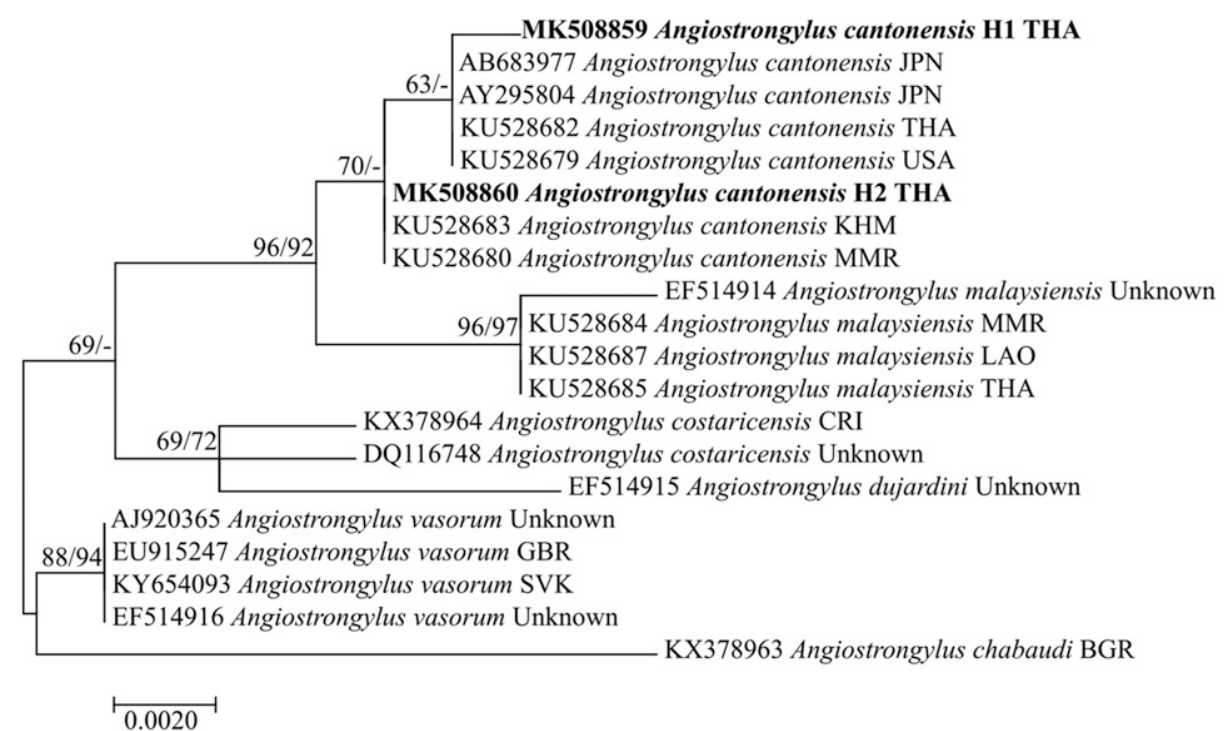

FIGURE 3. Phylogenetic relationships inferred from an alignment of small nuclear ribosomal subunit rRNA gene sequences using maximum likelihood. Sequences of Angiostrongylus species obtained from GenBank are indicated with the accession no. and the country code (ISO $3166-1$ alpha-3 code). Support values (ML bootstrap [percentages of 1,000 replicates]/Bayesian posterior probabilities) are shown above the branches. A dash (-) instead of a numerical support value indicates that a particular grouping was not found by that method of analysis. Sequences obtained in the present study are highlighted in bold. Scale bars indicate substitutions per nucleotide position. $\mathrm{H} 1$ and $\mathrm{H} 2$ represent patients 1 and 2 , respectively.

from our sequences consistently placed $A$. malaysiensis as a close sister to $A$. cantonensis.

\section{DISCUSSION}

The most confirmatory diagnostic method for optic neuritis caused by Angiostrongylus infection is to find larvae or juvenile worms by ophthalmoscopy. ${ }^{12}$ Morphological features have been generally used for the identification of Angiostrongylus species recovered from angiostrongyliasis patients. ${ }^{12,21,22}$ However, it is difficult for less-trained parasitologists to differentiate the causative species, and it will also be time consuming. ${ }^{23}$ Briefly, adults of $A$. cantonensis, $A$. mackerrasae, and A. malaysiensis can be distinguished from one another by the size of spicule and the arrangement of bursal rays of adult males, as well as the length of the vagina and of the tip of the tail in adult females. ${ }^{23}$ Angiostrongylus costaricensis recovered from human cases was identified as a distinct species based on morphological criteria. ${ }^{24,25}$ Fifth-stage larvae of the four species overlap in morphological features and are therefore difficult to identify by morphology. Molecular information, such as analysis of nucleotide sequences, is therefore a useful tool for the identification of Angiostrongylus to the species level but has rarely been applied to fifth-stage larvae. Fifth-stage larvae of Angiostrongylus cantonensis in Vietnam were identified using SSU ribosomal DNA sequences (99\% similarity with reference sequences in GenBank). ${ }^{13}$

Phylogenetic trees of the sequences (SSU rRNA, ITS2, and COI) obtained for four to six Angiostrongylus species (Figures 3-5) show that the Thai $A$. cantonensis in this study are closely

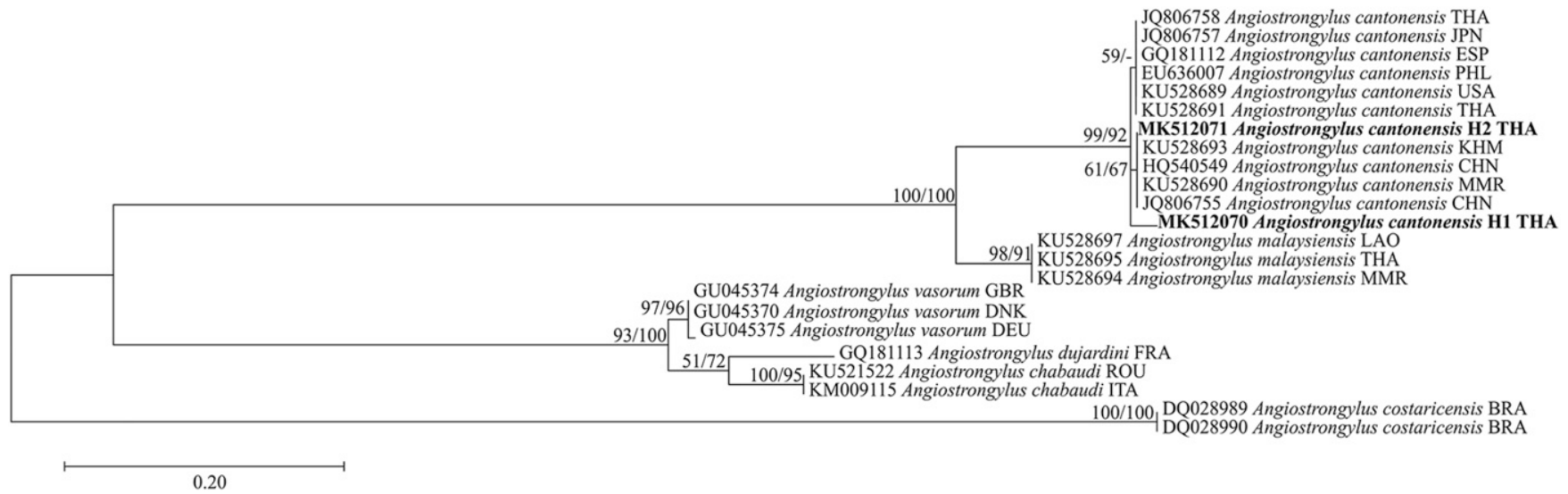

FIGURE 4. Phylogenetic relationships inferred from an alignment of ITS2 region sequences using maximum likelihood (ML). Sequences of Angiostrongylus species obtained from GenBank are indicated with the accession no. and the country code (ISO $3166-1$ alpha-3 code). Support values (ML bootstrap [percentages of 1,000 replicates]/Bayesian posterior probabilities) are shown above the branches. A dash (-) instead of a numerical support value indicates that a particular grouping was not found by that method of analysis. Sequences obtained in the present study are highlighted in bold. Scale bars indicate substitutions per nucleotide position. $\mathrm{H} 1$ and $\mathrm{H} 2$ represent patients 1 and 2 , respectively. 


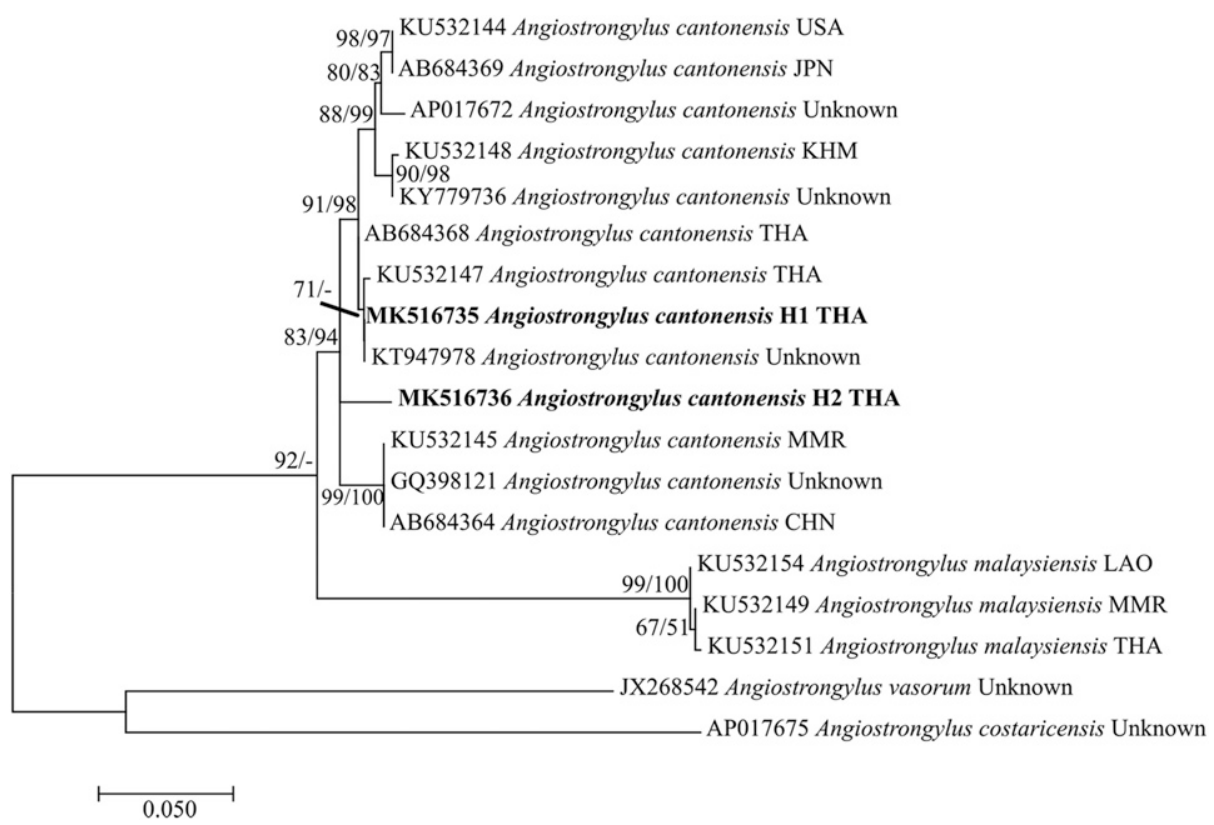

FIGURE 5. Phylogenetic relationships inferred from an alignment of $\mathrm{COI}$ gene sequences using maximum likelihood (ML). Sequences of Angiostrongylus species obtained from GenBank are indicated with the accession no. and the country code (ISO $3166-1$ alpha-3 code). Support values (ML bootstrap [percentages of 1,000 replicates]/Bayesian posterior probabilities) are shown above the branches. A dash (-) instead of a numerical support value indicates that a particular grouping was not found by that method of analysis. Sequences obtained in the present study are highlighted in bold. Scale bars indicate substitutions per nucleotide position. $\mathrm{H} 1$ and $\mathrm{H} 2$ represent patients 1 and 2, respectively.

related to other $A$. cantonensis populations for which sequences are available in GenBank (Figures 3-5); there is little sequence variation within the species. The extent of molecular variation and its relationship to geographical distribution and/or pathogenicity require further investigation. A close relationship between $A$. cantonensis and $A$. malaysiensis is apparent from the phylogenetic trees. A molecular survey of Angiostrongylus larvae in land snails found that these two species have broadly overlapping distributions in Lao PDR, Cambodia, Myanmar, and Thailand, ${ }^{17}$ but nobody has tried to confirm which species causes ocular disease in this region.

To our knowledge, the present study is the first to confirm by molecular analysis that $A$. cantonensis is a cause of angiostrongyliasis in Thailand. This was performed by sequencing DNA from fifth-stage larvae recovered from patients. DNA sequencing analysis can prevent misidentification based on the morphological features of Angiostrongylus species that cause human diseases, as previously reviewed. ${ }^{12}$ Here, we have described a method that is excellent for differential diagnosis of angiostrongyliasis. It is also important for epidemiological view.

Received September 20, 2019. Accepted for publication February 27, 2020.

Published online March 30, 2020.

Acknowledgment: We would like to thank David Blair for his valuable suggestions and assistance with the presentation of this manuscript through Khon Kaen University Publication Clinic, Thailand.

Financial support: This study was supported by Distinguished Research Professor Grant, Thailand Research Fund (P. M. I. and W. M., grant number DPG6280002); a Khon Kaen Univerisity Grant; a Scholarship under Doctoral Training Program from Graduate School Research Affairs and Khon Kaen University (L. S., grant number 60164).
Authors' addresses: Patcharaporn Boonroumkaew, Oranuch Sanpool, Lakkhana Sadaow, Pewpan M. Intapan, and Wanchai Maleewong, Department of Parasitology, Faculty of Medicine, Khon Kaen University, Khon Kaen, Thailand, and Research and Diagnostic Center for Emerging Infectious Diseases, Faculty of Medicine, Khon Kaen University, Khon Kaen, Thailand, E-mails: patcharapom.bo@kkumail.com, sanpoolor@yahoo.com, sadaow1986@gmail.com, pewpan@kku.ac.th, and wanch_ma@kku.ac.th. Thuss Sanguansak, Department of Ophthalmology, Faculty of Medicine, Khon Kaen University, Khon Kaen, Thailand, E-mail: t_sanguansak@hotmail.com. Siriraksa Visaetsilpanonta, Department of Ophthalmology, Retina Unit, Udon Thani Hospital, Udon Thani, Thailand, E-mail: trichromats@gmail.com.

\section{REFERENCES}

1. Robles Mdel R, Navone GT, Kinsella JM, 2008. A new angiostrongylid (Nematoda) species from the pulmonary arteries of Akodon azarae (Rodentia: Cricetidae) in Argentina. J Parasitol 94: 515-519.

2. Eamsobhana P, Lim PE, Yong HS, 2013. Genetic diversity of the rat lungworm, Angiostrongylus cantonensis, the major cause of eosinophilic meningitis. Hawaii J Med Public Health 72: 15-17.

3. Tsai HC et al., 2001. Eosinophilic meningitis caused by Angiostrongylus cantonensis: report of 17 cases. Am J Med 111: 109-114.

4. Tsai HC, Chen YS, Yen CM, 2013. Human parasitic meningitis caused by Angiostrongylus cantonensis infection in Taiwan. Hawaii J Med Public Health 72: 26-27.

5. Thiengo SC, Simoes Rde O, Fernandez MA, Maldonado A Jr, 2013. Angiostrongylus cantonensis and rat lungworm disease in Brazil. Hawaii J Med Public Health 72: 18-22.

6. Wang QP, Lai DH, Zhu XQ, Chen XG, Lun ZR, 2008. Human angiostrongyliasis. Lancet Infect Dis 8: 621-630.

7. Wan KS, Weng WC, 2004. Eosinophilic meningitis in a child raising snails as pets. Acta Trop 90: 51-53.

8. Kramer $\mathrm{MH}$ et al., 1998. First reported outbreak of abdominal angiostrongyliasis. Clin Infect Dis 26: 365-372.

9. Prociv P, Spratt DM, Carlisle MS, 2000. Neuro-angiostrongyliasis: unresolved issues. Int J Parasitol 30: 1295-1303.

10. Monte TC, Gentile R, Garcia J, Mota E, Santos JN, Maldonado Junior A, 2014. Brazilian Angiostrongylus cantonensis 
haplotypes, ac8 and ac9, have two different biological and morphological profiles. Mem Inst Oswaldo Cruz 109: 1057-1063.

11. Newton LA, Chilton NB, Beveridge I, Hoste H, Nansen $P$, Gasser RB, 1998. Genetic markers for strongylid nematodes of livestock defined by PCR-based restriction analysis of spacer rDNA. Acta Trop 69: 1-15.

12. Feng Y, Nawa Y, Sawanyavisuth K, Lv Z, Wu ZD, 2013. Comprehensive review of ocular angiostrongyliasis with special reference to optic neuritis. Korean J Parasitol 51: 613-619.

13. De NV, Duyet le V, Chai JY, 2015. A Case of ocular angiostrongyliasis with molecular identification of the species in Vietnam. Korean J Parasitol 53: 713-717.

14. Andrade GC, Dias JRO, Maia A, Kanecadan LA, Moraes NSB, Belfort Junior R, Lasiste JME, Burnier MN, 2018. Intravitreal Angiostrongylus cantonensis: first case report in South America. Arq Bras Oftalmol 81: 63-65.

15. Sinawat S, Trisakul T, Choi S, Morley M, Sinawat S, Yospaiboon Y, 2019. Ocular angiostrongyliasis in Thailand: a retrospective analysis over two decades. Clin Ophthalmol 13: 1027-1031.

16. Tokiwa T et al., 2012. Phylogenetic relationships of rat lungworm, Angiostrongylus cantonensis, isolated from different geographical regions revealed widespread multiple lineages. Parasitol Int 61: 431-436.

17. Rodpai R, Intapan PM, Thanchomnang T, Sanpool O, Sadaow L, Laymanivong S, Aung WP, Phosuk I, Laummaunwai P, Maleewong W, 2016. Angiostrongylus cantonensis and A. malaysiensis broadly overlap in Thailand, Lao PDR, Cambodia and Myanmar: a molecular survey of larvae in land snails. PLoS One 11: e0161128.
18. Hall TA, 1999. BioEdit: a user-friendly biological sequence alignment editor and analysis program for windows 95/98/NT. Nucleic Acids Symp Ser 41: 95-98.

19. Kumar S, Stecher G, Tamura K, 2016. MEGA7: molecular evolutionary genetics analysis version 7.0 for bigger datasets. $\mathrm{Mol}$ Biol Evol 33: 1870-1874.

20. Ronquist F, Teslenko M, van der Mark P, Ayres DL, Darling A, Höhna S, Larget B, Liu L, Suchard MA, Huelsenbeck JP, 2012. MrBayes 3.2: efficient Bayesian phylogenetic inference and model choice across a large model space. Syst Biol 61: 539-542.

21. Widagdo S, Lokollo DM, Margono SS, 1977. Ocular angiostrongyliasis in Semarang, Central Java. Am J Trop Med Hyg 26: 72-74.

22. Sinawat $S$, Sanguansak T, Angkawinijwong T, Ratanapakorn $T$, Intapan PM, Sinawat S, Yospaiboon Y, 2008. Ocular angiostrongyliasis: clinical study of three cases. Eye (Lond) 22: 1446-1448.

23. Bhaibulaya M, 1979. Morphology and taxonomy of major Angiostrongylus species of eastern Asia and Australia. Cross $\mathrm{JH}$, ed. Studies on angiostrongyliasis in Eastern Asia and Australia. Taipei, Taiwan: NAMRU-2 Special Publication (NAMRU2-SP-44), 4-25.

24. Morera P, Céspedes R, 1970. Angiostrongylus costaricensis n. $\mathrm{sp}$. (Nematoda: Metastrongyloidea), a new lungworm occurring in man in Costa Rica. Rev Biol Trop 18: 173-185.

25. Morera P,1973. Life history and redescription of Angiostrongylus costaricensis Morera and Céspedes, 1971. Am J Trop Med Hyg 22: 613-621. 